\title{
Effects of ABT-737 combined with irradiation treatment on uterine cervical cancer cells
}

\author{
HUANG-PIN SHEN ${ }^{1-3^{*}}$, WEN-JUN WU ${ }^{1 *}$, JIUNN-LIANG KO $^{1}$, TZU-FAN WU ${ }^{1}$, \\ SHUN-FA YANG ${ }^{1,4}$, CHIH-HSIEN WU ${ }^{1}$, CHIA-MING YEH ${ }^{1}$ and PO-HUI WANG ${ }^{1-3}$ \\ ${ }^{1}$ Institute of Medicine, Chung Shan Medical University; ${ }^{2}$ Department of Obstetrics and Gynecology, \\ Chung Shan Medical University Hospital; ${ }^{3}$ School of Medicine, Chung Shan Medical University; \\ ${ }^{4}$ Department of Medical Research, Chung Shan Medical University Hospital, Taichung 402, Taiwan, R.O.C.
}

Received July 9, 2018; Accepted April 24, 2019

DOI: 10.3892/ol.2019.10755

\begin{abstract}
The aim of the present study was to examine the role of ABT-737, an inhibitor of B-cell lymphoma 2 (Bcl-2), in enhancing the effect of irradiation on uterine cervical cancer. Based on The Cancer Genomic Atlas (TCGA), Bcl-2 mRNA expression was associated with the Tumor-Node-Metastasis stage of cervical cancer. Therefore, it was hypothesized that Bcl-2 inhibition may decrease the progression of cervical cancer. ABT-737 was added to irradiation treatment to evaluate its effectiveness in inhibiting cancer cell progression. $\mathrm{SiHa}$ and CaSki cervical cancer cells were selected for in vitro assays. Patients with advanced stage III uterine cancer had slightly increased mRNA expression levels of Bcl-2 compared with patients with stage I cancer, although the difference was not significant. ABT-737 and radiation administration induced a synergistic cytotoxic effect based on the MTT assay and flow cytometry results, where an increase in apoptosis was observed. The apoptotic percentages were significantly increased in the cells treated with a combination of ABT-737 and irradiation. Loss of mitochondrial membrane potential and gain of reactive oxygen species (ROS) were detected by
\end{abstract}

Correspondence to: Professor Po-Hui Wang, Institute of Medicine, Chung Shan Medical University, 110, Section 1, Chien-Kuo North Road, Taichung 402, Taiwan, R.O.C.

E-mail: wang082160@gmail.com

*Contributed equally

Abbreviations: ABT-737, 4-[4-[(4'-chloro[1,1'-biphenyl]-2-yl) meth yl]-1-piperazinyl]-N-[[4-[[(1R)-3-(dimethylamino)-1-[(phenylthio) methyl]propyl]amino]-3-nitrophenyl] sulfonyl]-benzamide; Bcl-2, B-cell lymphoma 2; BH3, Bcl-2 homology domain 3; MMP, mitochondrial membrane potential; JC-1, 5,5',6,6'-tetrachloro-1,1',3, 3'-tetraethylbenzimi-dazolylcarbocyanine iodide; PARP, poly ADP ribose polymerase; ROS, reactive oxygen species; TS, thymidylate synthase; VDAC1, voltage-dependent anion channel 1

Key words: ABT-73, B-cell lymphoma 2, irradiation, uterine cervical cancer, mitochondrial membrane potential, apoptosis flow cytometry in CaSki and SiHa cells treated with ABT-737 and radiation. Additionally, the protein expression levels of the cleaved forms of poly ADP ribose polymerase and caspase-7 were increased following the combined treatment. In conclusion, ABT-737 and irradiation may induce apoptosis via loss of mitochondrial membrane potential and a ROS-dependent apoptotic pathway in CaSki and $\mathrm{SiHa}$ cells. The present study indicates that ABT-737 may be a potential irradiation adjuvant when treating cervical cancer.

\section{Introduction}

We previously reported that the immunoreactivity of voltage-dependent anion channel 1 (VDAC1) is significantly increased in uterine cervical cancer tissues compared with that in normal tissues (1). Cell viability decreased following VDAC1 gene silencing in SiHa and CaSki cervical cancer cells. In addition, cancer tissues with increased VDAC1 immunoreactivity exhibited deep stromal invasion and an increase in the tumor size. The N-terminal region of VDAC1 is the target of an anti-apoptotic member of the B-cell lymphoma-2 (Bcl-2) family $(2,3)$. Bcl-2 may protect against the apoptosis of cervical cancer cells via its interaction with the $\mathrm{N}$-terminal part of VDAC1 (4). Therefore, it is hypothesized that Bcl-2 inhibition may reduce the progression of cervical cancer.

The Bcl-2 family consists of the pro-apoptotic members, $\mathrm{Bcl}-2$-associated $\mathrm{X}$ protein $(\mathrm{Bax})$ and $\mathrm{Bcl}-2$ homology domain 3 (BH3)-only proteins, in addition to the anti-apoptotic members, including Bcl-2, Bcl-xL and Bcl-w (5,6). ABT-737 is a well-characterized $\mathrm{BH} 3$ mimetic that activates the pro-apoptotic $\mathrm{Bcl}-2$ like protein (7). As an inhibitor of $\mathrm{Bcl}-2$, ABT-737 predominately interacts with $\mathrm{Bcl}-2$ and further enhances the effect of apoptosis by activating pro-apoptotic proteins, including $\mathrm{Bcl}-2$-associated death (Bad) promoter, and therefore induces cell death mostly through the intrinsic pathway of apoptosis (7-9).

A previous study showed the efficacy of ABT-737 administration in vivo by itself (10); however, a number of preclinical investigations demonstrated the effectiveness of ABT-737 in conjunction with chemotherapy and radiotherapy (11-13). ABT-737 was an effective adjuvant to radiotherapy in head and neck squamous cell carcinoma (14). 
Uterine cervical cancer is the second most common type of gynecological cancer in Taiwan, based on the 2013 annual cancer registry report. In Taiwanese women in 2013, cervical cancer was the seventh most common cancer, with 1,579 cases, and was also ranked seventh with regard to the number of cancer-associated mortalities (15). Radiotherapy is a cornerstone of treatment of cervical cancer, especially for the locally advanced stages (16). To the best of our knowledge, there is only one study that has reported the effect of combining ABT-737 and irradiation on cervical cancers (17). ABT-737 may improve the radiation sensitivity of cervical cancer HeLa cells and thereby promote apoptosis (17). Histologically, HeLa cells are of adenocarcinoma cell histology. However, the majority of cervical cancer types present with a squamous cell carcinoma (SCC) histology. Therefore, the present study was conducted to elucidate the combined effect of ABT-737 and irradiation on SCC uterine cervix cancer cells using the SiHa and CaSki cell lines, and to evaluate whether ABT-737 could strengthen the effect of irradiation on cervical cancer cells.

\section{Materials and methods}

The cancer genome atlas (TCGA). Based on the cervical cancer data from The Cancer Genome Atlas (18) (https://tcga-data.nci. nih.gov/tcga/), which corresponds to the cervical squamous cell carcinoma and endocervical adenocarcinoma (CESC) dataset $(n=286)$ from the Broad GDAC Firehose (http://gdac. broadinstitute.org/). Scatter plots of the expression values were generated with respect to the pathological tumor stage for $\mathrm{Bcl}-2$ using Prism software (GraphPad Prism, version 6.0, GraphPad Software). The Bcl-2 expression of patients with advanced stage was compared with that of patients with stage I cancer. TCGA was used to determine whether an association existed between uterine cervical cancer Tumor-Node-Metastasis stage (19) and Bcl-2 expression. The present study was approved by The Institutional Review Board of Chung Shan Medical University Hospital (Taichung, Taiwan).

Cell culture. Human uterine cervical cancer CaSki and SiHa cell lines were purchased from The American Type Culture Collection. SiHa cells were cultured in Dulbecco's modified Eagle's medium (Gibco; Thermo Fisher Scientific, Inc.), and CaSki cells were cultured in RPMI-1640 medium (Gibco; Thermo Fisher Scientific, Inc.). All media were supplemented with $2 \mathrm{mM}$ glutamine, $100 \mu \mathrm{M}$ sodium pyruvate, $100 \mu \mathrm{M}$ non-essential amino acids, $1 \%$ penicillin-streptomycin and $10 \%$ fetal bovine serum (Gibco; Thermo Fisher Scientific, Inc.). Cells were grown in a humidified atmosphere with $5 \% \mathrm{CO}_{2}$ at $37^{\circ} \mathrm{C}$.

Cell viability assay. Cell viability was examined by an MTT assay. In total $\sim 5 \times 10^{3}$ of CaSki or SiHa cells were seeded per well in a 96-well plate and cultured for 4 days. MTT was added into each well to a final concentration of $0.5 \mathrm{mg} / \mathrm{ml}$. The insoluble formazan was collected and dissolved in dimethylsulfoxide, and the optical density value was measured with a scanning spectrophotometer at a wavelength of $570 \mathrm{~nm}$.

Mitochondrial membrane potential (MMP) assay. In total, $\sim 5 \times 10^{5} \mathrm{CaSki}$ or SiHa cells were seeded in 6-cm dishes and treated with ABT-737 (2.5 or 5.0 $\mu \mathrm{M}$ ) (Cayman Chemical
Company) combined with irradiation (10 or $20 \mathrm{~Gy}$ ) for $48 \mathrm{~h}$. Untreated control was defined as ABT-737 $0 \mu \mathrm{M}$ and irradiation $0 \mathrm{~Gy}$. At $30 \mathrm{~min}$ prior to harvesting, the cells were stained at $37^{\circ} \mathrm{C}$ with a $2.5-\mu \mathrm{M}$ final concentration of 5,5,6,6'-tetrachloro-1,1,3,3'-tetraethylbenzimi-dazolylcarbocyanine iodide (JC-1) dye (Invitrogen; Thermo Fisher Scientific, Inc.) to detect the MMP by fluorescence microscopy and flow cytometry using CellQuest 5.1 software (BD Biosciences). Membrane-permeant JC-1 dye is widely used in apoptosis study to monitor MMP and can be used as an indicator of MMP in various cell types $(20,21)$. Changes in MMP were assessed by the intensity of red and green fluorescence signals detected by flow cytometry. Red fluorescence [light-emitting material (lem), $590 \mathrm{~nm}$ ] indicated JC-1 aggregation in the mitochondria with an increased MMP in healthy cells, whereas green fluorescence (lem, $527 \mathrm{~nm}$ ) indicated JC-1 monomers in the cytoplasm with a decreased MMP in apoptotic cells (22).

Irradiation treatment. $\mathrm{CaSki}$ or $\mathrm{SiHa}$ cells were plated at a density of $5 \times 10^{5}$ cells per dish in 6 -cm dishes. After $16 \mathrm{~h}$ of incubation at $37^{\circ} \mathrm{C}$ to allow sufficient time for the cells to completely adhere to the surface, the cells were treated with radiation at doses of 10 or 20 Gy by Elekta Axesse ${ }^{\mathrm{TM}}$ instrument.

Western blotting. Cells were lysed in a buffer containing $50 \mathrm{mM}$ Tris (pH 7.4), $150 \mathrm{mM} \mathrm{NaCl}, 2 \mathrm{mM}$ EDTA, $1 \mathrm{mM}$ $\mathrm{Na}_{3} \mathrm{VO}_{4}, 10 \mathrm{mM} \mathrm{NaF}, 10 \mathrm{mg} / \mathrm{ml}$ aprotinin, $10 \mathrm{mg} / \mathrm{ml}$ leupeptin, $1 \mathrm{mM}$ phenylmethylsulfonyl fluoride and $1 \%$ Triton-100. The protein concentrations were determined using Bio-Rad Protein assay (Bio-Rad Laboratories, Inc.). Equal amounts of protein $(20 \mu \mathrm{g})$ were subjected to gel electrophoresis on a $10 \%$ gel and then transferred to polyvinylidene difluoride membranes. Blots were incubated in a Tris-buffered saline solution at $\mathrm{pH} 7.6$ containing 5\% skimmed dry milk and $0.1 \%$ (v/v) Tween-20 for $1 \mathrm{~h}$ at $25^{\circ} \mathrm{C}$. The membranes were incubated overnight at $4^{\circ} \mathrm{C}$ with primary antibodies, washed with PBS-Tween-20, and incubated with horseradish peroxidase-conjugated anti-rabbit secondary antibody (cat. no. 7074; dilution 1:5,000; Cell Signaling Technology, Inc.) for $1 \mathrm{~h}$ at room temperature. An enhanced chemiluminescence kit (PerkinElmer, Inc.) was used to detect the target proteins. Primary antibodies against $\beta$-actin (cat. no. 3700; dilution 1:5,000), Poly ADP ribose polymerase (PARP; cat. no. 9542; dilution 1:1,000), cleaved caspase-7 (cat. no. 9491; dilution 1:500), retinoblastoma (RB; cat. no. 9309; dilution 1:1,000) and phosphorylated RB (pRB; cat. no. 9308; dilution 1:1,000) were purchased from Cell Signaling Technology, Inc. Thymidylate synthase (TS; cat. no. 113289; dilution 1:1,000) and cyclin-dependent kinase 6 (CDK6; cat. no. 103992; dilution 1:1,000) were purchased from GeneTex, Inc.

Reactive oxygen species (ROS) detection. Cell-permeant 2',7'-dichlorodihydrofluorescein diacetate $\left(\mathrm{H}_{2}\right.$ DCFDA; Invitrogen Thermo Fisher Scientific, Inc.) is a widely used ROS indicator. The reduced non-fluorescent fluorescein $\mathrm{H}_{2}$ DCFDA can be oxidized and converted into fluorescent 2',7'-dichlorofluorescein (DCF) by intracellular ROS. $\mathrm{H}_{2}$ DCFDA $(10 \mu \mathrm{M})$ was used to label intracellular ROS at $4^{\circ} \mathrm{C}$ for $30 \mathrm{~min}$ and the DCF intensity was detected by flow cytometry. 
Analysis of cell cycle and apoptosis. Cells were treated with or without ABT-737/radiation for $48 \mathrm{~h}$, and the cell cycle distribution was analyzed using flow cytometry. A total of $5 \times 10^{5}$ cells were trypsinized, washed with PBS and fixed in $80 \%$ ethanol at $4^{\circ} \mathrm{C}$ for $30 \mathrm{~min}$, then washed with PBS and incubated with $100 \mu \mathrm{g} / \mathrm{ml}$ RNase A (Sigma-Aldrich; Merck KGaA) at $37^{\circ} \mathrm{C}$ for $30 \mathrm{~min}$, prior to being stained with propidium iodide (PI; $50 \mu \mathrm{g} / \mathrm{ml}$; Sigma-Aldrich; Merck KGaA) at $37^{\circ} \mathrm{C}$ for $10 \mathrm{~min}$, and analyzed using flow cytometry on a BD FACSCalibur flow cytometer (BD Biosciences). The percentage of cells in different phases of the cell cycle was analyzed using Cell-FIT software version 2.0 (Becton Dickinson Instruments). Apoptosis in CaSki and SiHA cells was confirmed by BD FACSCalibur flow cytometry to detect phosphatidylserine expression on cell surfaces by using fluorescein isothiocyanate (FITC)-labeled Annexin V and PI (FITC-Annexin V Apoptosis Detection kit I; BD Pharmingen ${ }^{\mathrm{TM}}$; BD Biosciences).

Statistical analysis. Data were analyzed by an analysis of variance followed by Dunnett's post hoc test using Predictive Analytics software (version 18; IBM Corp.) to evaluate the significance of differences between the untreated control group and the other groups. Data are presented as the mean \pm standard deviation based on three independent repeats. $\mathrm{P}<0.05$ was considered to indicate a statistically significant difference.

\section{Results}

Comparison of Bcl-2 expression among cancer tissues of different stages. Based on TCGA data, the mRNA expression levels of Bcl-2 among cancer tissues of different stages was compared. There was no significant difference in expression levels between the stages (Fig. 1).

Effect of ABT-737 and irradiation on cell viability in CaSki and SiHa uterine cervical cells. CaSki and SiHa cells were treated with different concentrations of $\mathrm{Bcl}-2$ inhibitor ABT-737 $(0,1,2.5,5$, and $10 \mu \mathrm{M})$ and different doses of irradiation $(0,10$, and $20 \mathrm{~Gy})$ for $48 \mathrm{~h}$. Cell viability was analyzed by an MTT assay. As presented in Fig. 2, the cell viability of the CaSki and SiHa cells was significantly reduced following treatment with all doses of ABT-737 and at all levels of irradiation compared with the untreated control (ABT-737 $0 \mu \mathrm{M}$ and irradiation $0 \mathrm{~Gy}$ ). The effect of treatment and irradiation on cell viability was dose-dependent and there was an enhanced effect on cell viability when cells were treated with ABT-737 and irradiation synergistically.

Effect of ABT-737 combined with irradiation on MMP in CaSki and SiHa cells. CaSki and SiHa cells were treated with different concentrations of ABT-737 and different doses of irradiation for $48 \mathrm{~h}$. The cells were stained with JC-1 dye to detect the presence of MMP by both fluorescent microscopy and flow cytometry. The live cells stained with JC-1 fluoresced red, whereas the apoptotic cells fluoresced green. The number of cells showing green fluorescence as detected by fluorescent microscope increased in CaSki and SiHa cells treated with ABT-737 and irradiation compared with untreated control (ABT-737 $0 \mu \mathrm{M}$ and irradiation $0 \mathrm{~Gy}$ ) (Fig. 3A and C). The effect was more evident in CaSki cells than in SiHa cells.

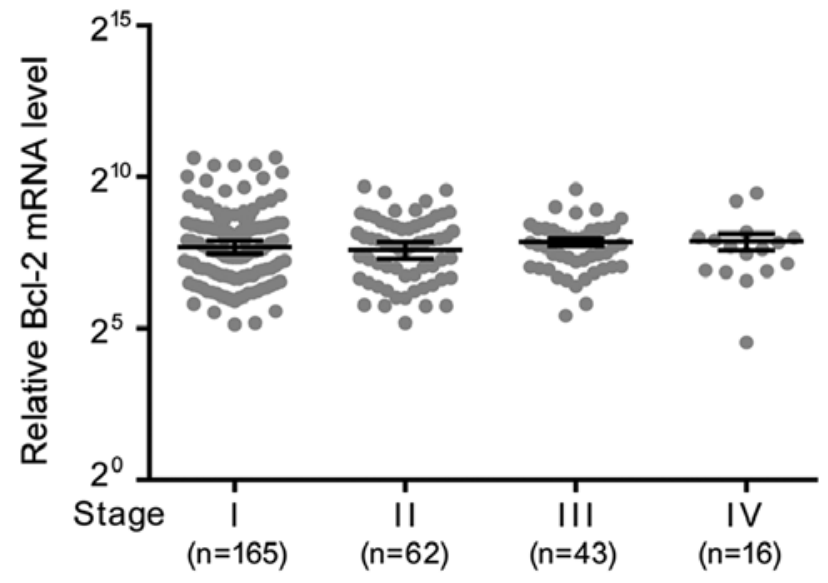

Figure 1. mRNA expression levels of Bcl-2 among cancer tissues of the uterine cervix in stages I-IV based on The Cancer Genome Atlas data. There was no significant association between Bcl-2 expression levels and cervical cancer stage. Bcl-2, B-cell lymphoma 2 .

Similar results were shown in Fig. 3B detected by flow cytometry. The results found at $20 \mathrm{~Gy}$ and $5 \mu \mathrm{M}$ do not appear to follow the same pattern of increase in the SiHa cell line (Fig. 3D). Potential reasons for this could be due to the treated SiHa cells being dead for too long a time and breaking up into cell debris or they were not lysed adequately enough to be detected by flow cytometry. So the increased pattern was not observed. R2 represents live cells, whereas R1 or R3 represent apoptotic cells. These results indicate that the MMP was decreased in CaSki and SiHa cells treated with ABT-737 and irradiation.

Effect of ABT-737 and irradiation on cell apoptosis in CaSki and SiHa cells. CaSki and SiHa cells were treated with different concentrations of Bcl-2 inhibitor ABT-737 $(2.5$ and $5 \mu \mathrm{M})$ and different doses of irradiation (10 and $20 \mathrm{~Gy}$ ) for $48 \mathrm{~h}$. The cells were stained with Annexin V-FITC and PI to detect cell apoptosis by flow cytometry. Annexin V-FITC-positive (early apoptosis, right lower panel) and Annexin V-FITC/PI-positive (late apoptosis, right upper panel) were quantified as apoptotic cells. The percentage of apoptotic cells increased significantly in CaSki and SiHa cells treated with ABT-737 and irradiation (Fig. 4A and B). However, the apoptotic percentage in SiHa cells treated with ABT-737 $5 \mu \mathrm{M}$ and irradiation $20 \mathrm{~Gy}$ was less compared with the cells treated with $5 \mu \mathrm{M}$ ABT-737 and 10 Gy irradiation. Potential reasons for this are discussed above, including prolonged cell death or improper lysing. Additionally, 10 Gy irradiation may be a toxicity threshold of SiHa cells. As such, the increased apoptotic pattern was not observed. The increase in apoptosis was evident in cells treated with ABT-737 and irradiation. Apoptosis was also determined by the presence of cleaved caspase- 7 by western blotting. When ABT-737 concentration and irradiation dose were increased, the cleaved bands of PARP and caspase-7 became visible (Fig. 4C and D). These results demonstrate that ABT-737 and irradiation could enhance cell apoptosis in CaSki and SiHa cells.

Effect of ABT-737 combined with irradiation on ROS production in CaSki and SiHa cells. Following treatment of 

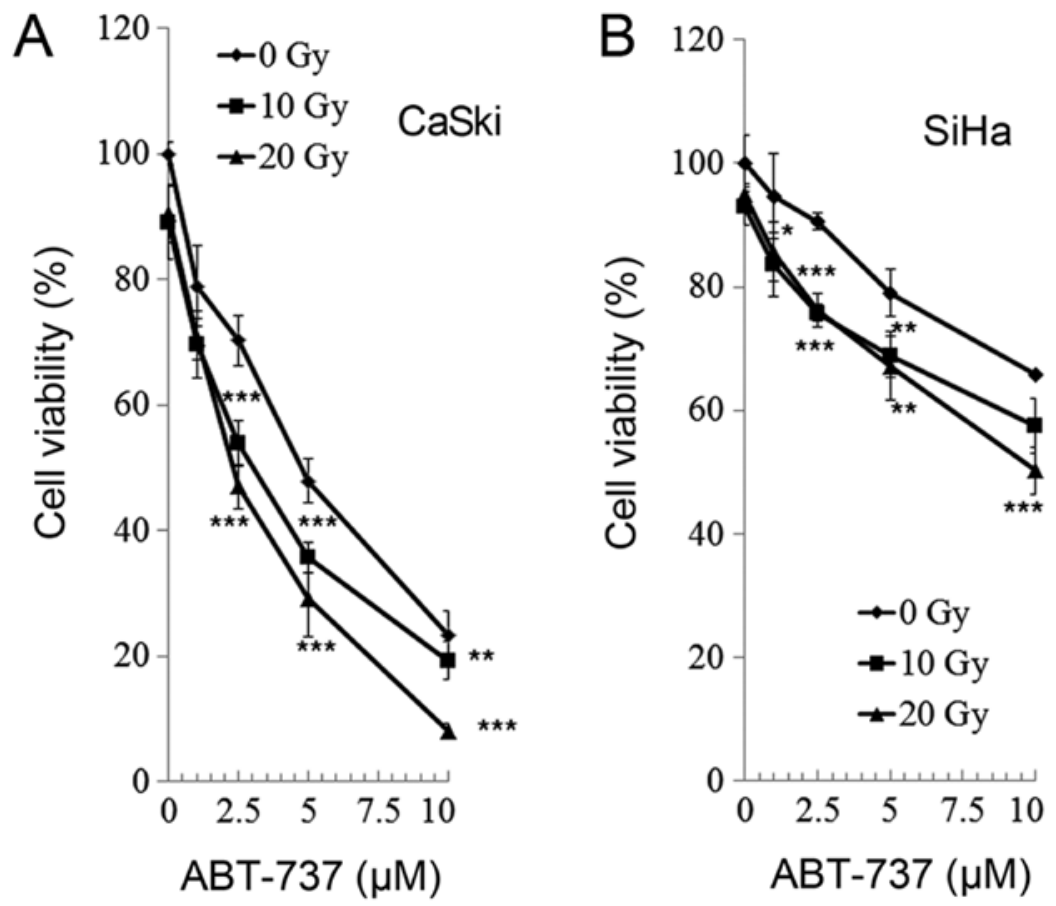

Figure 2. Effect of ABT-737 and irradiation on cell viability in CaSki and SiHa uterine cervical cancer cells after 48 h. (A) CaSki and (B) SiHa cells were treated with different concentrations of ABT-737 and radiation for $48 \mathrm{~h}$. Cell viability was analyzed by an MTT assay. Significant differences vs. untreated control (ABT-737 $0 \mu \mathrm{M} /$ irradiation $0 \mathrm{~Gy}$ ) were determined by Dunnett's test and are indicated by ${ }^{*} \mathrm{P}<0.05,{ }^{* *} \mathrm{P}<0.01$ and ${ }^{* * *} \mathrm{P}<0.001$.

cells with ABT-737 and irradiation, the cells were stained with $\mathrm{H}_{2}$ DCFDA dye to detect ROS by flow cytometry. As shown in Fig. 5, the augmented ROS production caused by ABT-737 and irradiation was more notable in CaSki cells than in SiHa cells.

Effect of ABT-737 and irradiation on the cell cycle and phosphorylation of $\mathrm{RB}$ in CaSki and SiHa cells. The cell cycle was arrested in the $\mathrm{G}_{2} / \mathrm{M}$ phase when CaSki and SiHa cells were treated with irradiation only; however, the percentage of cells in the sub- $\mathrm{G}_{1}$ phase was amplified in CaSki and $\mathrm{SiHa}$ cells co-treated with ABT-737 and irradiation (Fig. 6A and B). Western blotting results demonstrated that $\mathrm{pRB}$ protein expression was upregulated in CaSki cells treated with irradiation alone but was downregulated in the cells treated with ABT-737. The protein expression levels of CDK6 were increased, but those of TS were decreased in CaSki cells co-treated with ABT-737 and irradiation (Fig. 6C). The protein expression levels of pRB, CDK6 and TS were not affected in SiHa cells treated with ABT-737 and irradiation (Fig. 6D).

\section{Discussion}

Although there was no significant association between Bcl-2 mRNA level and the stage of cervical cancer, the Bcl-2 protein expression level in cervical cancer tissues was significantly higher compared with in the normal cervix, chronic cervicitis or cervical intraepithelial neoplasia (23). Bcl-2 level was positively correlated with clinical stage (23). In addition, the number of malignant lesions expressing Bcl-2 protein was higher compared with premalignant lesions (24). In cervical intraepithelial neoplasia lesions, Bcl-2 expression increased as the severity of the cancer, measured by the grade, increased (24). Based on these studies, the mechanistic effects of inhibiting Bcl-2 and irradiating cervical cancer cells were investigated. To the best of our knowledge, there are no reports demonstrating the effects of combined Bcl-2 inhibition and irradiation on cervical cancer. In the present study, a target strategy was proposed to improve patient prognosis via Bcl-2 inhibition, and the effect of combined Bcl-2 inhibition and irradiation on cervical cancer cells was evaluated. As an inhibitor of Bcl-2, ABT-737 is one of the best-characterized BH3 mimetics that activates pro-apoptotic Bcl-2-like protein 11 (BIM) $(8,25)$. Activation of BIM is associated with markers associated with a favorable overall survival prognosis for patients with cervical cancer (26). Therefore, the possible mechanisms of Bcl-2 inhibition and the enhanced effect of combined Bcl-2 inhibitor and irradiation treatment in reducing cancer cell progression were investigated.

The activity of the Bcl-2 family members is mediated through its interaction with the mitochondria, especially with VDAC1, the outer mitochondrial membrane (OMM) transporter, to regulate mitochondria-mediated apoptosis, frequently referred to as the intrinsic pathway, by regulating OMM permeability (6,27). ABT-737 enhances the effect of apoptosis by activating pro-apoptotic proteins such as BIM, and therefore induces cell death primarily through mitochondria-mediated apoptosis (4,7-9). Sugiyama et al (28) demonstrated that BIM interacts with VDAC, and this interaction was enhanced during apoptosis. The present study demonstrated that ABT-737 and irradiation induced apoptosis in cervical cancer CaSki and SiHa cells. The MMP decreased following treatment with increased doses of irradiation and ABT-737, particularly in the CaSki cells. Upon stimulation of apoptosis, mitochondrial permeability transition occurred 
A

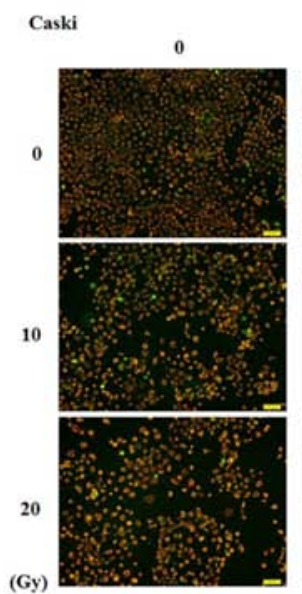

C

SiHa

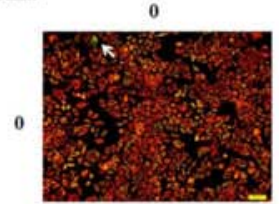

10

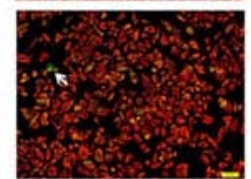

20

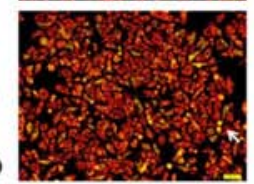

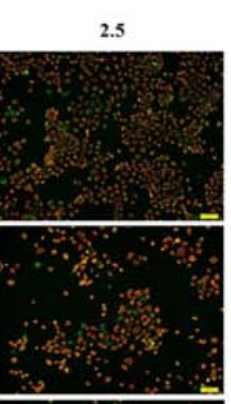
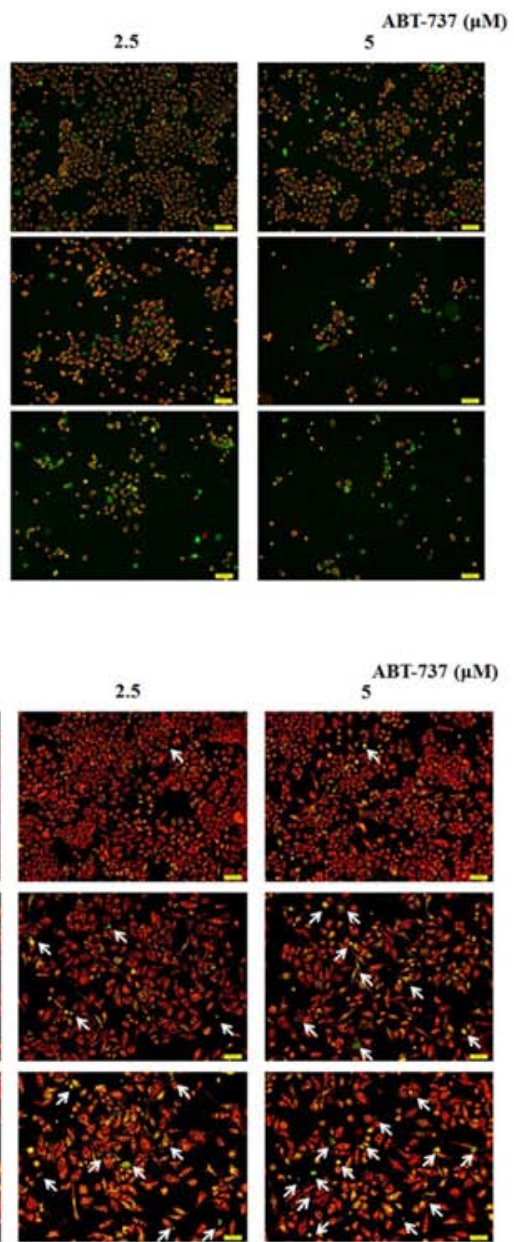
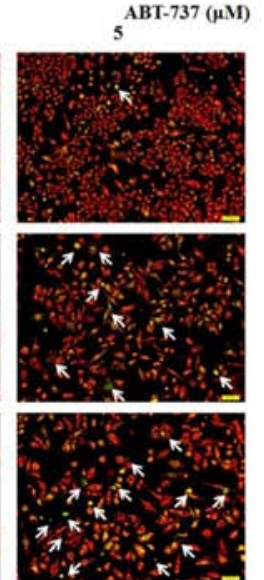

B
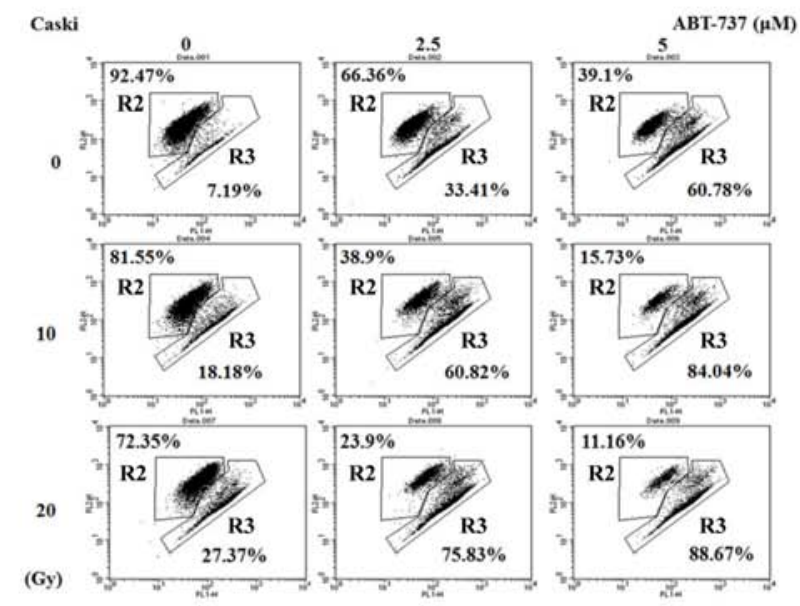

D
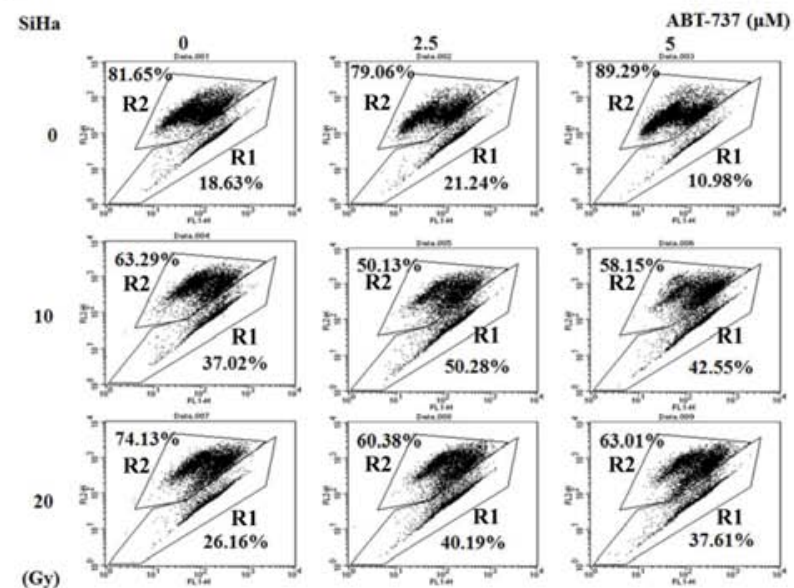

Figure 3. Effect of ABT-737 combined with irradiation on the MMP in CaSki and SiHa cells. (A) Fluorescent images and (B) flow cytometry of CaSki cells treated with ABT-737. (C) Fluorescent images and (D) flow cytometry of CaSki cells treated with ABT-737. Cells were stained with JC-1 dye to detect the MMP by (A and C) fluorescence microscopy and (B and D) flow cytometry. Changes in MMP were assessed by the intensity of red fluorescence (R2) and green fluorescence (R1 or R3). The arrows signify the apoptotic cells that show green fluorescence. Results are representative of three independent experiments. MMP, mitochondrial membrane potential; FL, fluorescence.

via the mitochondrial permeability transition pore, which is composed of VDAC at the OMM, adenine nucleotide at the inner mitochondrial membrane and cyclophilin $\mathrm{D}$ in the mitochondrial matrix, after which, the MMP collapsed (29,30). The proteins in the mitochondrial intermembrane space, such as cytochrome $c$, are released when a cell undergoes apoptosis. The data presented in the present study suggest that ABT-737 and radiation may exert a synergistic apoptotic effect on cervical cancer cell lines. Bcl-2 protects against the apoptosis of cervical cancer cells via its interaction with the N-terminal part of VDAC1 (4). There is potential for ABT-737 to bind to Bcl-2 with a high affinity and disrupt its interaction with VDAC1, resulting in loss of the MMP and enhanced apoptosis. Therefore, ABT-737 and irradiation may induce apoptosis via the loss of MMP caused by the interaction of VDAC1 with pro-apoptotic proteins mediated by a ROS-dependent intrinsic pathway in CaSki and SiHa cells. In previous studies, ABT-737 also improved the efficiency of radiotherapy and chemotherapy in breast cancer (31) and non-small cell lung cancer cell lines (32), and it may induce the autophagy of prostate cancer via releasing Beclin-1 (33).
In the present study, ABT-737 increased ROS production and enhanced the apoptotic effect of irradiation in CaSki cells. Increased levels of mitochondrial ROS initiate intrinsic apoptosis, leading to the release of mitochondrial apoptogenic factors such as cytochrome $c$, an apoptosis-inducing factor, into the cytosol (34). ABT-737 and irradiation may induce apoptosis via the ROS-dependent mitochondria-mediated apoptosis pathway. A limitation of the present study was that Bcl-2 and cytochrome $c$ expression were not measured. ABT-737 has been demonstrated to bind to Bcl-2 with a high affinity and disrupts the interaction with the pro-apoptotic proteins, Bax/Bak, thus enhancing apoptosis. Bax and Bak homodimers promote apoptosis via pore formation within the mitochondria, leading to permeabilization of the mitochondrial outer membrane, release of cytochrome $c$ and activation of the caspase cascade (35). Hepatocellular carcinoma cells with increased levels of Bcl-2 may resist the effect of ABT-737 by activation of the ROS-Janus kinase-autophagy pathway (36). In the present study, ABT-737 enhanced the apoptotic effect of irradiation on the loss of the MMP in cervical cancer CaSki and SiHa cells. The cancer cells may protect themselves from 
A

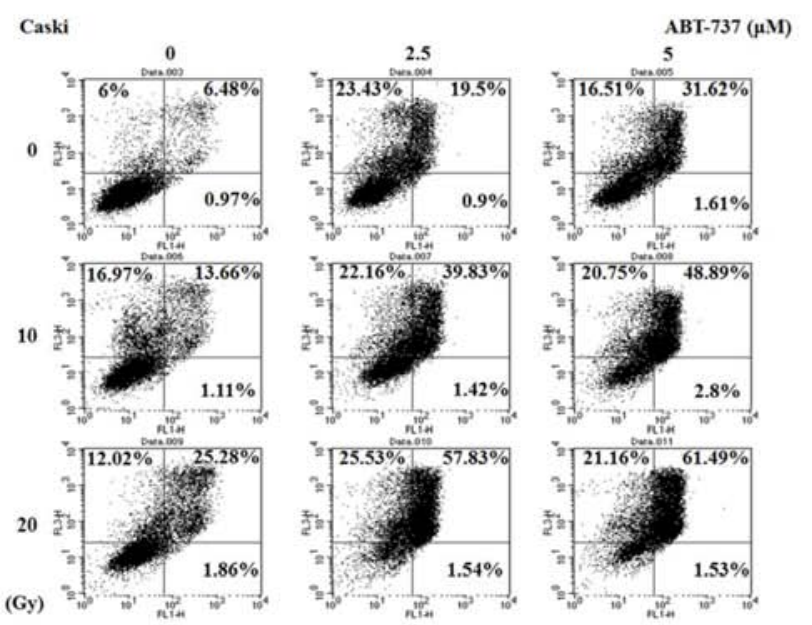

B
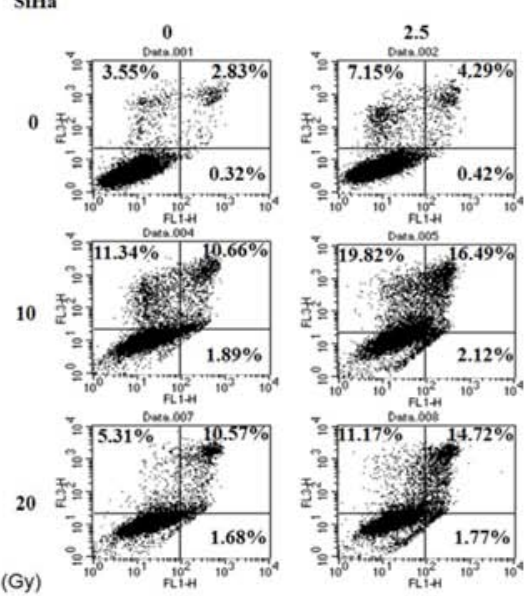
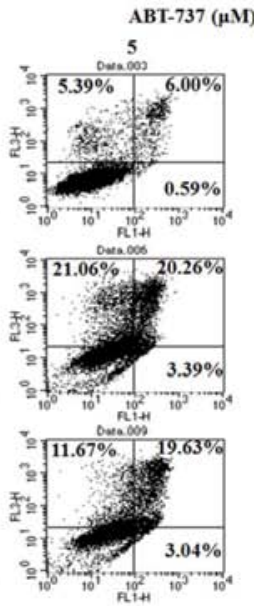

C

Caski
D

SiHa

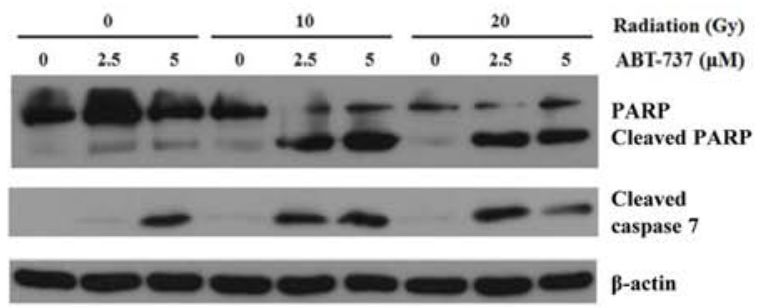

Figure 4. Effect of ABT-737 and irradiation on apoptosis in CaSki and SiHa cells. (A) CaSki and (B) SiHa cells were treated with ABT-737 combined with irradiation for $48 \mathrm{~h}$. Cells were stained by Annexin V-FITC and PI. Annexin V-FITC positive (early apoptosis) and Annexin V-FITC/PI positive (late apoptosis) were quantified as apoptotic cells. (C) CaSki and (D) SiHa cells were treated with ABT-737 combined with irradiation for $48 \mathrm{~h}$. Cleaved PARP and cleaved caspase-7 were detected by western blotting. $\beta$-actin was used as a loading control. FITC, fluorescein isothiocyanate; PI, propidium iodide; PARP, poly ADP ribose polymerase; FL, fluorescence.
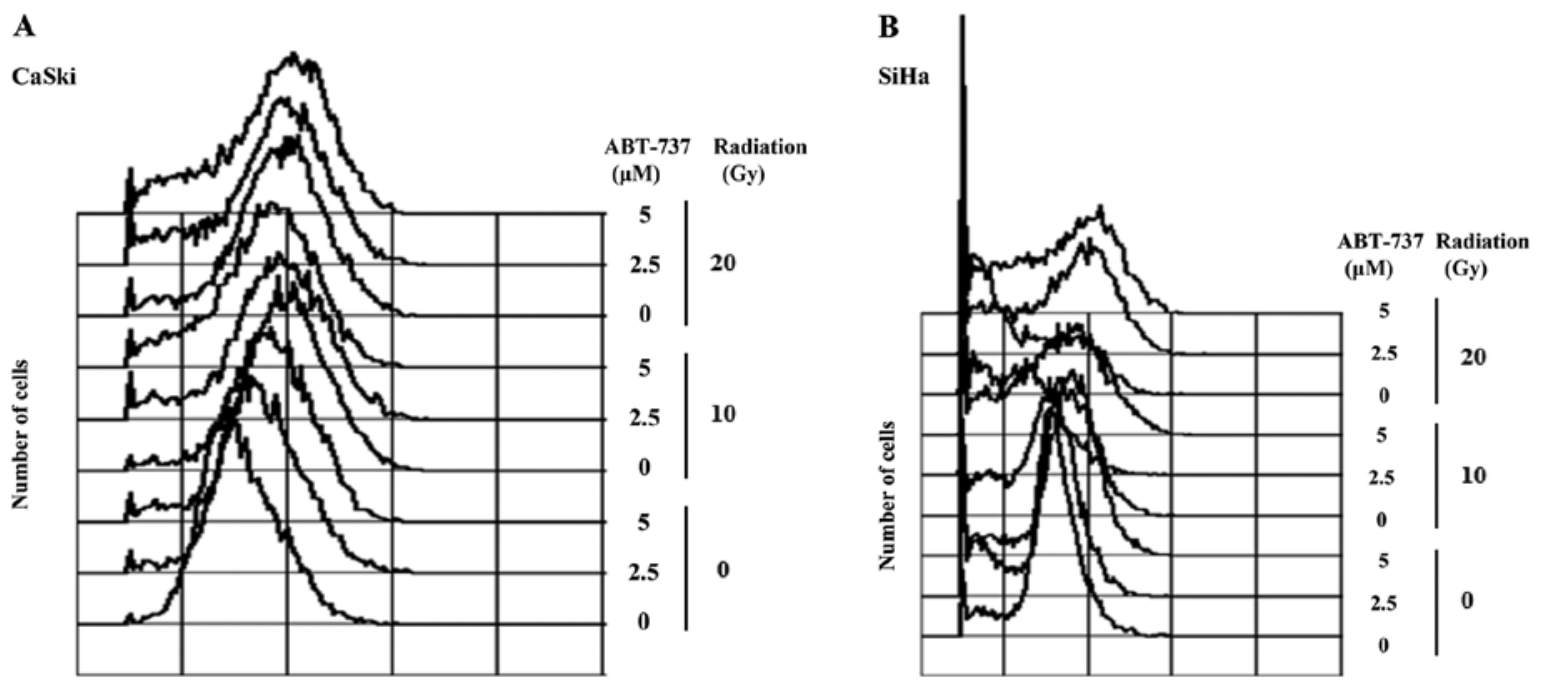

Figure 5. Effect of ABT-737 combined with irradiation on ROS production in CaSki and SiHa cells. (A) CaSki and (B) SiHa cells were treated with ABT-737 combined with irradiation for $48 \mathrm{~h}$. Cells were stained with 2',7'-dichlorodihydrofluorescein diacetate dye to detect ROS by flow cytometry. Results are representative of three independent experiments. ROS, reactive oxygen species.

cell apoptosis via autophagy to remove the damaged mitochondria (37,38). However, experiments to determine whether autophagy was induced when cells were treated with ABT-737 and irradiation were inconclusive (data not shown).
RB functions to prevent uncontrolled cell growth by arresting cell cycle progression until a cell is ready to divide. At this point, RB is phosphorylated and becomes inactive, thus allowing the cell cycle to progress. Based on the results 
A

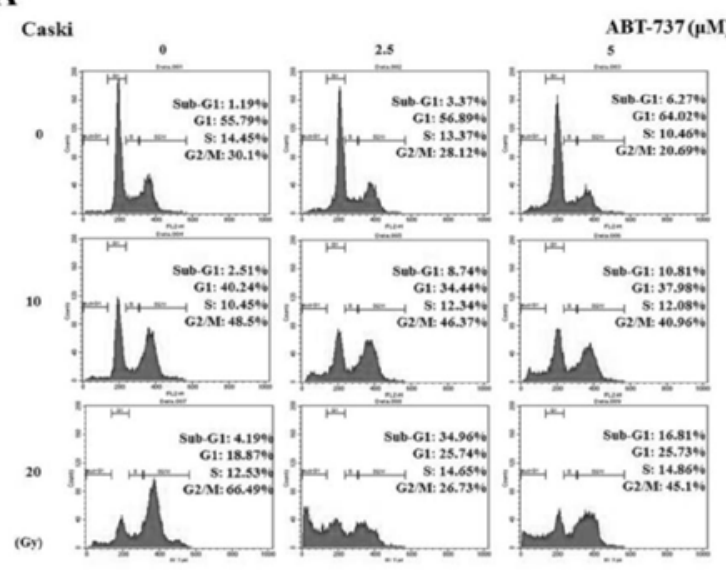

C

Caski
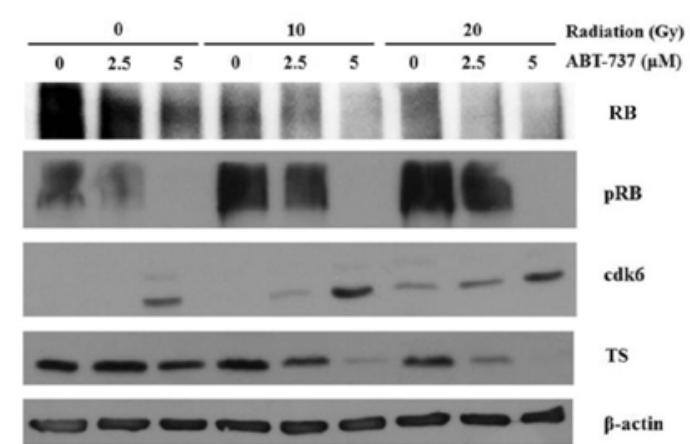

B

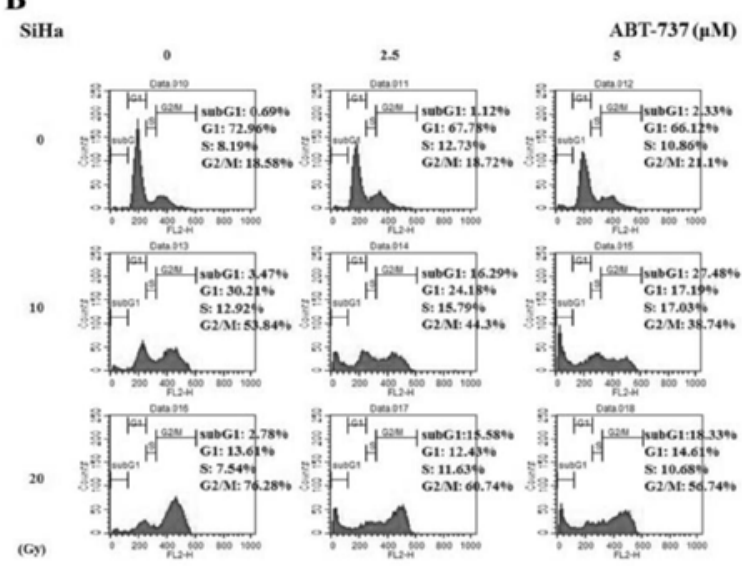

D

SiHa

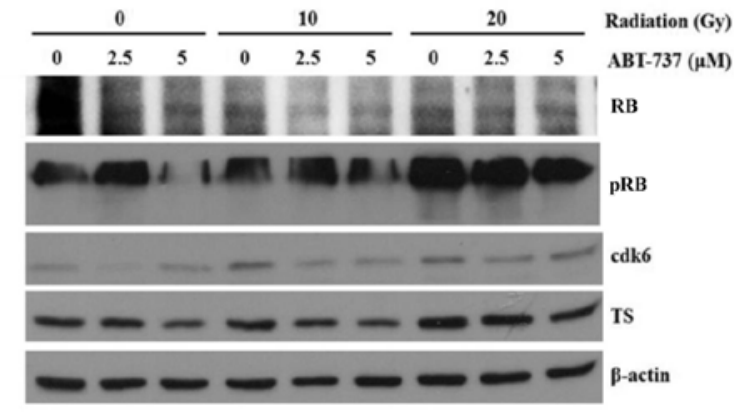

Figure 6. Effect of ABT-737 and irradiation on the cell cycle in CaSki and SiHa cells. (A) CaSki and (B) SiHa cells were treated by ABT-737 combined with irradiation for $48 \mathrm{~h}$. The cells were stained with propidium iodide dye to detect the cell cycle by flow cytometry. (C) CaSki and (D) SiHa cells were treated with ABT-737 combined with irradiation for 48 h. pRB, RB, CDK6 and TS protein expression were assessed by western blot. $\beta$-actin was a loading control. $\mathrm{RB}$, retinoblastoma; p, phosphorylated; CDK6, cyclin-dependent kinase-6; TS, thymidylate synthase; FL, fluorescence.

of the present study, ABT-737 may potentiate the irradiation effect to decrease cell viability by promoting or inducing apoptosis. ABT-737 and radiation treatment decreased pRB expression in the CaSki cells. TS serves a vital role in early DNA biosynthesis (39). Healthy DNA synthesis and insertion of required for the normal functions of the body and in order to avoid cancerous activity. Furthermore, the synthesis of important nucleotides must be inhibited for cell growth. Therefore, TS has become an important target for cancer treatment through chemotherapy or radiotherapy. In the present study, the protein expression levels of TS were decreased in CaSki cells co-treated with ABT-737 and radiation. The sensitivity of TS to ABT-737 and irradiation may provide another application for treating patients with cervical cancer. CDK6 in conjunction with CDK4 acts as a switch that initially appears in $\mathrm{G}_{1}$ and subsequently directs the cell toward the S phase of the cell cycle (40). By studying a DNA damage-induced senescence model of human fibroblasts, Brookes et al (41) demonstrated an unexpected role for CDK4 in contributing to a $\mathrm{G}_{2} / \mathrm{M}$ cell cycle arrest and the senescent phenotype. The findings of the present study may explain that ABT-737, especially when combined with irradiation, increases the expression of CDK6 and consequently induces $\mathrm{a} \mathrm{G}_{2} / \mathrm{M}$ cell cycle arrest.

The apoptotic effects were conflicting when CaSki and $\mathrm{SiHa}$ cells were treated with ABT-737 and irradiation. It is possible that different methods of detecting apoptosis may result in different sensitivities. A cell cycle study may not be sufficient to demonstrate apoptosis. Cell-cycle analysis would reveal the fragmentation of DNA (sub- $\mathrm{G}_{1}$ ) that occurs in the late stage of apoptosis, and which may occur during necrosis as well. Therefore, the number of cells in the sub- $\mathrm{G}_{1}$ phase is usually lower than that reported by other assays that detect both early and late stages of apoptosis.

Radiotherapy is the cornerstone of cervical cancer treatment, especially for the locally advanced stages (16). ABT-737 can improve the sensitivity of HeLa cervical cancer cells to irradiation and thus, induce cell apoptosis (17). However, the HeLa cancer cells are of an adenocarcinoma histological type. CaSki and $\mathrm{SiHa}$ cells are derived from $\mathrm{SCC}$ of the uterine cervix. When CaSki and SiHa cells were subjected to ABT-737 and irradiation treatment, the CaSki cells became more sensitive than $\mathrm{SiHa}$ cells based on the results of the in vitro assays, which may have been due to the levels of antioxidant enzymes or HPV 16 copy number in each cell type. Filippova et al (42) reported that $\mathrm{SiHa}$ cells are more resistant to doxorubicin and cisplatin treatment, and express higher levels of antioxidant enzymes than CaSki cells. These different profiles may have contributed to their different responses to treatments with chemotherapy. SiHa cells contain 1 to 2 copies of the HPV 16 genome per cell, whereas CaSki cells contain $\sim 600$ copies per cell (43). Upon 
treatment of these two cell lines with TNF at varying concentrations, a viability analysis revealed that the CaSki cells with a higher HPV 16 level were sensitive to the cytokine, whereas SiHa cells with only 1 to 2 copies of the genome were relatively resistant (44). This indicated that certain aspects of the high number of HPV 16 copies in the CaSki genome, such as the increased amount of one or more viral proteins, may contribute to its TNF sensitivity (44).

In conclusion, the present study highlighted the potential implication of the combinatorial approach for treating cervical cancer cells with both a Bcl-2 inhibitor and radiation therapy. The percentages of cells in the sub- $G_{1}$ phase were amplified in CaSki and SiHa cells co-treated with ABT-737 and irradiation. ABT-737 may enhance the induction of apoptosis in human cervical SCC via loss of the MMP and an ROS-dependent intrinsic apoptosis pathway in CaSki and $\mathrm{SiHa}$ cells. Although the CDK6 expression was increased, the reduced expression levels of $\mathrm{pRB}$ and TS indicate the subsequent $\mathrm{G}_{1} / \mathrm{S}$ checkpoint regulation of cell progression in CaSki cells was abrogated. Based on these findings, therapeutic strategies should use ABT-737 as a target to induce cell apoptosis, reduce cell growth and progression, and enhance the therapeutic efficacy of radiation therapy. Therefore, ABT-737 may be a potential irradiation adjuvant for treating patients with cervical cancer.

\section{Acknowledgements}

Not applicable.

\section{Funding}

The present study was funded by The Taiwan Ministry of Science and Technology (grant no. MOST 105-2314-B-040016-MY2) and Chung Shan Medical University Hospital (grant no. CSH-2017-D-002).

\section{Availability of data and materials}

The datasets used and/or analyzed during the present study are available from the author on reasonable request.

\section{Authors' contributions}

PW and HS conceived and designed the study. WW and JK performed experiments and analyzed the data. TW, SY, CW and CY conducted data analysis and interpretation. PW wrote the manuscript. WW edited and revised the manuscript. All authors discussed results/discussion and collaborated in drafting the manuscript.

\section{Ethics approval and consent to participate}

The present study was approved by The Institutional Review Board of Chung Shan Medical University Hospital (Taichung, Taiwan).

\section{Patient consent for publication}

Not applicable.

\section{Competing interests}

The authors declare that they have no competing interests.

\section{References}

1. Wu CH, Lin YW, Wu TF, Ko JL and Wang PH: Clinical implication of voltage-dependent anion channel 1 in uterine cervical cancer and its action on cervical cancer cells. Oncotarget 7: 4210-4225, 2016.

2. Abu-Hamad S, Arbel N, Calo D, Arzoine L, Israelson A, Keinan N, Ben-Romano R, Friedman O and Shoshan-Barmatz V: The VDAC1 N-terminus is essential both for apoptosis and the protective effect of anti-apoptotic proteins. J Cell Sci 122: 1906-1916, 2009.

3. Arbel N and Shoshan-Barmatz V: Voltage-dependent anion channel 1-based peptides interact with $\mathrm{Bcl}-2$ to prevent antiapoptotic activity. J Biol Chem 285: 6053-6062, 2010

4. Shoshan-Barmatz V, De Pinto V, Zweckstetter M, Raviv Z, Keinan N and Arbel N: VDAC, a multi-functional mitochondrial protein regulating cell life and death. Mol Aspects Med 31: 227-285, 2010.

5. Adams JM and Cory S: The Bcl-2 apoptotic switch in cancer development and therapy. Oncogene 26: 1324-1337, 2007.

6. Youle RJ and Strasser A: The BCL-2 protein family: Opposing activities that mediate cell death. Nat Rev Mol Cell Biol 9: 47-59, 2008.

7. Del Gaizo Moore V, Brown JR, Certo M, Love TM, Novina CD and Letai A: Chronic lymphocytic leukemia requires BCL2 to sequester prodeath BIM, explaining sensitivity to BCL2 antagonist ABT-737. J Clin Invest 117: 112-121, 2007.

8. Oltersdorf T, Elmore SW, Shoemaker AR, Armstrong RC, Augeri DJ, Belli BA, Bruncko M, Deckwerth TL, Dinges J, Hajduk PJ, et al: An inhibitor of Bcl-2 family proteins induces regression of solid tumours. Nature 435: 677-681, 2005.

9. Chen S, Dai Y, Harada H, Dent P and Grant S: Mcl-1 down-regulation potentiates ABT-737 lethality by cooperatively inducing Bak activation and Bax translocation. Cancer Res 67: 782-791, 2007.

10. Mason KD, Vandenberg CJ, Scott CL, Wei AH, Cory S, Huang DC and Roberts AW: In vivo efficacy of the Bcl-2 antagonist ABT-737 against aggressive Myc-driven lymphomas. Proc Natl Acad Sci USA 105: 17961-17966, 2008.

11. Hann CL, Daniel VC, Sugar EA, Dobromilskaya I, Murphy SC, Cope L, Lin X, Hierman JS, Wilburn DL, Watkins DN and Rudin CM: Therapeutic efficacy of ABT-737, a selective inhibitor of BCL-2, in small cell lung cancer. Cancer Res 68: 2321-2328, 2008.

12. Kutuk $\mathrm{O}$ and Letai A: Alteration of the mitochondrial apoptotic pathway is key to acquired paclitaxel resistance and can be reversed by ABT-737. Cancer Res 68: 7985-7994, 2008.

13. Tagscherer KE, Fassl A, Campos B, Farhadi M, Kraemer A, Böck BC, Macher-Goeppinger S, Radlwimmer B, Wiestler OD, Herold-Mende C and Roth W: Apoptosis-based treatment of glioblastomas with ABT-737, a novel small molecule inhibitor of Bcl-2 family proteins. Oncogene 27: 6646-6656, 2008.

14. Gilormini M, Malesys C, Armandy E, Manas P, Guy JB, Magné N, Rodriguez-Lafrasse C and Ardail D: Prefer ntial targeting of cancer stem cells in the radiosensitizing effect of ABT-737 on HNSCC. Oncotarget 7: 16731-16744, 2016.

15. Taiwan Cancer Registry Annual Report: Taiwan: Health promotion administration, ministry of health and welfare. https://www. hpa.gov.tw/File/Attach/5191/File_6166.pdf.

16. Marth C, Landoni F, Mahner S, McCormack M, Gonzalez-Martin A and Colombo N; ESMO Guidelines Committee: Cervical cancer: ESMO clinical practice guidelines for diagnosis, treatment and follow-up. Ann Oncol 28 (Suppl 4): iv72-iv83, 2017.

17. Wang H, Yang YB, Shen HM, Gu J, Li T and Li XM: ABT-737 induces Bim expression via JNK signaling pathway and its effect on the radiation sensitivity of HeLa cells. PLoS One 7: e52483, 2012.

18. Tomczak K, Czerwińska P and Wiznerowicz M: The cancer genome atlas (TCGA): An immeasurable source of knowledge. Contemp Oncol (Pozn) 19: A68-A77, 2015.

19. Pecorelli S: Revised FIGO staging for carcinoma of the vulva, cervix, and endometrium. Int J Gynecol Obstet 105: 103-104, 2009. 
20. Reers M, Smiley ST, Mottola-Hartshorn C, Chen A, Lin M and Chen LB: Mitochondrial membrane potential monitored by JC-1 dye. Methods Enzymol 260: 406-417, 1995

21. Elefantova K, Lakatos B, Kubickova J, Sulova Z and Breier A: Detection of the mitochondrial membrane potential by the cationic dye JC-1 in L1210 cells with massive overexpression of the plasma membrane ABCB1 drug transporter. Int J Mol Sci 19: pii: E1985, 2018

22. Shah BP, Pasquale N, De G, Tan T, Ma J and Lee KB: Core-shell nanoparticle-based peptide therapeutics and combined hyperthermia for enhanced cancer cell apoptosis. ACS Nano 8: 9379-9387, 2014

23. Zhou XL and Wang M: Expression levels of survivin, Bcl-2, and KAI1 proteins in cervical cancer and their correlation with metastasis. Genet Mol Res 14: 17059-17067, 2015.

24. Kamaraddi S, Nayak A, Honnappa S and Swarup A: Expression of Bcl-2 marker in premalignant lesions of cervical cancer. Int J Reprod Contracept Obstet Gyneco 15: 965-969, 2016.

25. Fulda S, Galluzzi L and Kroemer G: Targeting mitochondria for cancer therapy. Nat Rev Drug Discov 9: 447-464, 2010.

26. Kim BW, Cho H, Ylaya K, Kitano H, Chung JY, Hewitt SM and Kim JH: Bcl-2-like Protein 11 (BIM) expression is associated with favorable prognosis for patients with cervical cancer. Anticancer Res 37: 4873-4879, 2017.

27. Shoshan-Barmatz V, Zakar M, Rosenthal K and Abu-Hamad S: Key regions of VDAC1 functioning in apoptosis induction and regulation by hexokinase. Biochim Biophys Acta 1787: 421-430, 2009.

28. Sugiyama T, Shimizu S, Matsuoka Y, Yoneda Y and Tsujimoto Y: Activation of mitochondrial voltage-dependent anion channel by apro-apoptotic BH3-only protein Bim. Oncogene 21: 4944-4956, 2002.

29. Kroemer G, Galluzzi L and Brenner C: Mitochondrial membrane permeabilization in cell death. Physiol Rev 87: 99-163, 2007.

30. Halestrap AP and Richardson AP: The mitochondrial permeability transition: A current perspective on its identity and role in ischaemia/reperfusion injury. J Mol Cell Cardiol 78: 129-141, 2015.

31. Li JY, Li YY, Jin W, Yang Q, Shao ZM and Tian XS: ABT-737 reverses the acquired radioresistance of breast cancer cells by targeting Bcl-2 and Bcl-xL. J Exp Clin Cancer Res 31: 102, 2012

32. Kim KW, Moretti L, Mitchell LR, Jung DK and Lu B: Combined $\mathrm{Bcl}-2 / \mathrm{mammalian}$ target of rapamycin inhibition leads to enhanced radiosensitization via induction of apoptosis and autophagy in non-small cell lung tumor xenograft model. Clin Cancer Res 15: 6096-6105, 2009.
33. Lian J, Wu X, He F, Karnak D, Tang W, Meng Y, Xiang D, Ji M, Lawrence TS and Xu L: A natural BH3 mimetic induces autophagy in apoptosis-resistant prostate cancer via modulating Bcl-2-Beclin1 interaction at endoplasmic reticulum. Cell Death Differ 18: 60-71, 2011.

34. Redza-Dutordoir M and Averill-Bate DA: Activation of apoptosis signalling pathways by reactive oxygen species. Biochim Biophys Acta 1863: 2977-2992, 2016.

35. Chipuk JE, Moldoveanu T, Lambi F, Parsons MJ and Green DR The BCL-2 family reunion. Mol Cell 37: 299-310, 2010.

36. Ni Z, Wang B, Dai X, Ding W, Yang T, Li X, Lewin S, Xu L, Lian J and He F: HCC cells with high levels of Bcl-2 are resistant to ABT-737 via activation of the ROS-JNK-autophagy pathway. Free Radic Biol Med 70: 194-203, 2014.

37. Das DN, Naik PP, Mukhopadhyay S, Panda PK, Sinha N, Meher BR and Bhutia SK: Elimination of dysfunctional mitochondria through mitophagy suppresses benzo[a]pyrene-induced apoptosis. Free Radic Biol Med 112: 452-463, 2017.

38. Swiader A, Nahapetyan H, Faccini J, D'Angelo R, Mucher E, Elbaz M, Boya $\mathrm{P}$ and Vindis C: Mitophagy acts as a safeguard mechanism against human vascular smooth muscle cell apoptosis induced by atherogenic lipids. Oncotarget 7: 28821-28835, 2016.

39. Peters GJ, Backus HH, Freemantle S, van Triest B, CodacciPisanelli G, van der Wilt CL, Smid K, Lunec J, Calvert AH, Marsh S, et al: Induction of thymidylate synthase as a 5-fluorouracil resistance mechanism. Biochim Biophys Acta 1587: 194-205, 2002

40. Bertoli C, Skotheim JM and de Bruin RA: Control of cell cycle transcription during G1 and S phases. Nat Rev Mol Cell Biol 14: 518-528, 2013.

41. Brookes S, Gagrica S, Sanij E, Rowe J, Gregory FJ, Hara E and Peters G: Evidence for a CDK4-dependent checkpoint in a conditional model of cellular senescence. Cell Cycle 14: 1164-1173, 2015.

42. Filippova M, Filippov V, Williams VM, Zhang K, Kokoza A Bashkirova S and Duerksen-Hughes P: Cellular levels of oxidative stress affect the response of cervical cancer cells to chemotherapeutic agents. Biomed Res Int 2014: 574659, 2014.

43. ATCC: CRL-1550, HTB-35, 2005. http://www.atcc.org.

44. Filippova M, Brown-Bryan TA, Casiano CA and DuerksenHughes PJ: The human papillomavirus 16 E6 protein can either protect or further sensitize cells to TNF: Effect of dose. Cell Death Differ 12: 1622-1635, 2005. 